J. Chosun Natural Sci.

Vol. 7, No. 1 (2014) pp. $1-4$

http://dx.doi.org/10.13160/ricns.2014.7.1.1

\title{
Fabrication and Characterization of Free-Standing DBR Porous Silicon Film
}

\author{
Sungyong Um and Honglae Sohn ${ }^{\dagger}$
}

\begin{abstract}
Distributed Bragg reflector porous silicon of different characteristics were formed to determine their optical constants in the visible wavelength range using a periodic square wave current between low and high current densities. The surface and cross-sectional SEM images of distributed Bragg reflector porous silicon were obtained using a cold field emission scanning electron microscope. The surface image of distributed Bragg reflector porous silicon indicates that the distributions of pores are even. The cross-sectional image illustrates that the multilayer of distributed Bragg reflector porous silicon exhibits a depth of few microns and applying of square current density during the etching process results two distinct refractive indices in the contrast. Distributed Bragg reflector porous silicon exhibited a porosity depth profile that related directly to the current-time profile used in etch. Its free-standing film was obtained by applying an electropolishing current.
\end{abstract}

Key words: DBR, Refractive Index, Porous Silicon

\section{Introduction}

Micrometer-sized device containing one-dimensional photonic structure that can detect chemical compounds could be useful for a variety of applications such as chemical and biological sensors, environmental monitoring, or medical diagnostic. Porous silicon (PSi) is a very attractive material for refractive index fabrication because of its easiness in changing the refractive index. A lot of studies have been made on one- and twodimensional refractive index lattice structures, including studies on photonic crystals. PSi has a strong advantage such as high surface area, convenient surface chemistry, and optical signal transduction capability ${ }^{[1]}$. The high surface area and unique optical properties of porous silicon have been investigated for a variety of chemical and biological sensing applications ${ }^{[2]}$. Electrochemical etch of single crystalline silicon can easily control the pore morphology, thickness, and porosity by appropriate adjustment of the preparation conditions. In addition, chemical modification of porous silicon exhibits the modification of its physical, chemical, and electronic

Department of Chemistry, Chosun University, Gwangju 501-759, Korea

†Corresponding author: hsohn@chosun.ac.kr

(Received : January 10, 2014, Revised : February 15, 2014,

Accepted : February 20, 2014) properties $^{[3-10]}$. Rugate structured porous silicon having photonic structure of Rugate filter in which refractive index varies sinusoidally has been recently developed by applying a computer-generated pseudo-sinusoidal current waveform chemical or bio molecule can be detected based on changes in the spectral interference pattern. Distributed Bragg reflector porous silicon (DBR PSi) has been also developed and typically prepared by an applying a square current waveform to the etch cell which results two distinct indices and exhibits photonic structure of Bragg filters ${ }^{[11-16]}$. The processes to fabricate porous silicon two-dimensional refractive index lattices with Bragg structure in the vertical direction have been proposed.

\section{Experimental Section}

\subsection{Preparation of DBR PSi}

DBR PSi samples were prepared by an electrochemical etch of heavily doped $\mathrm{p}^{++}$-type silicon wafer (boron doped, polished on the $<100>$ face, resistivity of 0.8-1.2 $\mathrm{m} \Omega \cdot \mathrm{cm}^{-2}$, Siltronix, Inc.). The etching solution consisted of a $3: 1$ volume mixture of aqueous $48 \%$ hydrofluoric acid (ACS reagent, Aldrich Chemicals) and absolute ethanol (ACS reagent, Aldrich Chemicals). Galvanostatic etch was carried out in a Teflon cell applying 20 
cycles of a two-electrode configuration and performed in a dark room.DBR PSi was prepared by using a periodic pseudo-square wave current between $5 \mathrm{~mA} \cdot \mathrm{cm}^{-2}$ for $100 \mathrm{~s}$ and $50 \mathrm{~mA} \cdot \mathrm{cm}^{-2}$ for $3 \mathrm{~s}$. All samples were then rinsed several times with ethanol and dried under argon atmosphere prior to use. The resulting DBR PSi were removed from the silicon substrate by an applying of electropolishing current at $460 \mathrm{~mA} \cdot \mathrm{cm}^{2}$ for $100 \mathrm{~s}$ in a solution of $48 \%$ aqueous HF and ethanol (3:1 by volume) and $29 \mathrm{~mA} \cdot \mathrm{cm}^{-2}$ for $200 \mathrm{~s}$ in a solution of $48 \%$ aqueous $\mathrm{HF}$ and ethanol (1:15 by volume) to obtain a free-standing DBR PSi film.

\subsection{Instruments and Data Acquisitions}

The morphology of DBR PSi was observed with cold field emission scanning electron microscope (FE-SEM, S-4800, Hitachi). Interferometric reflectance spectra were recorded by using an Ocean Optics S2000 spectrometer fitted with a bifurcated fiber optic probe. Spectra were recorded with a CCD detector in the wavelength range $400 \sim 200 \mathrm{~nm}$. The illumination of the surface as well as the measurement of the reflected light was performed along an axis coincident with the surface normal.

\section{Results and Discussion}

DBR PSi whose layers of different porosity can be etched one on top of another makes PSi a very promising material for the development of dielectric multilayers that behave as interference filters for use in optoelectronics and displays a high reflectivity band with a Bragg wavelength $\lambda_{\text {Bragg }}$, depending on the thickness of the porous layers $\left(d_{1}, d_{2}\right)$ and the corresponding refractive indices of their porous layer $\left(n_{1}, n_{2}\right)$. The $m^{\text {th }}$ order of the Bragg reflection is given by:

$$
m \lambda_{\text {Bragg }}=2\left(d_{1} \cdot n_{1}+d_{2} \cdot n_{2}\right)
$$

High reflective DBR PSi in a specific narrow spectral region can be prepared by applying two different applied current densities. From the optical point of view, porous silicon can be described as a homogeneous mixture of silicon and air.), There is a constant absorption region in a wavelength range from about 1000 to $2500 \mathrm{~nm}$ where the c-Si substrate has a low extinction coefficient. If $n=$ const., the refractive indices were derived as follows:

$$
n=\frac{\lambda^{2}}{2 d \Delta \lambda} \text { or } n=\frac{1}{2 d \Delta v}
$$

Here, $v$ is the wavenumber and $v=1 / \lambda$. The bandgap widening of PSi due to quantum confinement effects leads to a decrease of the extinction coefficient $\mathrm{k}$, making PSi transparent in the whole infrared (IR) region. Interference filters in the IR have been demonstrated with good spectral behavior due to the low absorption of PS in this wavelength range. However, from the near infrared to the optical range (300-1000 nm), PSi has a strong extinction coefficient and dispersion. Due to a large distribution of pores sizes present in PSi, optical absorption is a function of the wavelength. For the development of such interference filters, it is first necessary to precisely determine the complex refractive index of the individual PSi layers, and to correlate them with the electrochemical formation parameters. However, since porous silicon is a dispersive and absorbing material, determination of both the real part (refractive index, $n$ ) and imaginary part (extinction coefficient, $k$ ) of the complex refractive index is not trivial. In the present work, PSi layers of different characteristics were formed to determine their optical constants in the visible wavelength range. Fig. 1 shows the reflectance spectra of two different PSi samples prepared with different current densities. One (red) whose reflection appears at around $600 \mathrm{~nm}$ is prepared with a low applied current density for 100 seconds of electrochemical etching and the other (blue) whose reflection appears at around 600 $\mathrm{nm}$ is prepared with a high applied current density for 5 seconds of electrochemical etching.

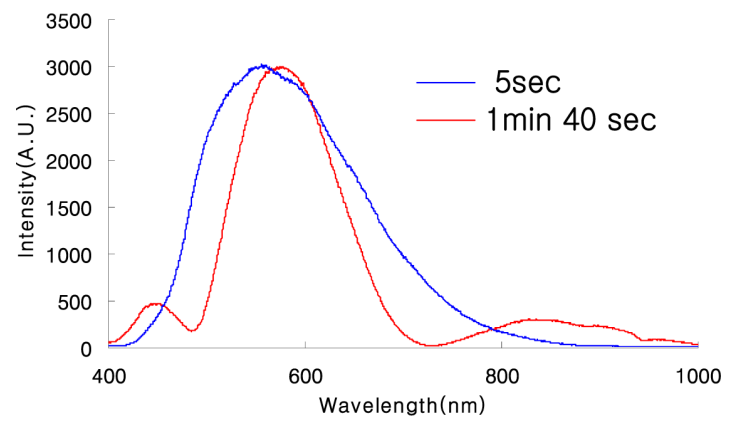

Fig. 1. Reflectance spectra of two different PSi samples prepared with a low current density (red) and a high current density (blue). 


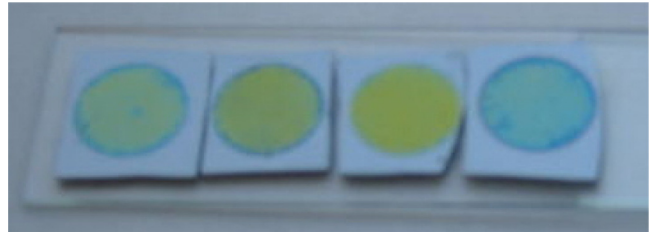

Fig. 2. Photograph of PSi samples prepared with a low current density.

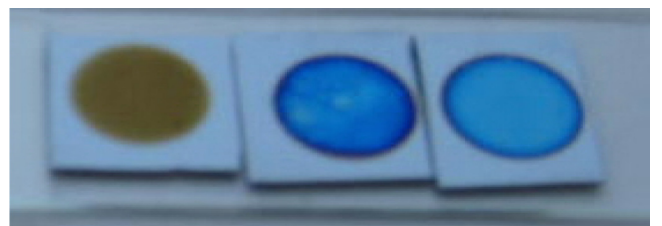

Fig. 3. Photograph of PSi samples a high current density.

Fig. 2 and 3 show the photographs of PSi samples which were prepared with a low current density (Fig. 2) and a high current density (Fig. 3). different PSi samples prepared with different current densities.

Typical etch parameters for DBR PSi structure involves using a periodic square wave current between low and high current densities. The surface and crosssectional SEM images shown in Fig. 4 and 5 were obtained using a cold field emission scanning electron microscope. DBR PSi displays a single reflection peak. The surface image of DBR PSi shown in Fig.4 indicates that the distributions of pores in DBR PSi are even. The cross-sectional image of DBR PSi shown in Fig. 5 illustrates that the multilayer of DBR PSi exhibits a depth of few microns and applying of square current density during the etching process results two distinct refractive indices in the contrast. DBR PSi exhibited a porosity depth profile that related directly to the current-time profile used in etch.

DBR PSi films have been removed from the silicon wafer by applying an electropolishing current. Fig. 6 shows the schematic diagram to obtain free-standing DBR PSi. Fig. 7 showed the reflection bands of DBR

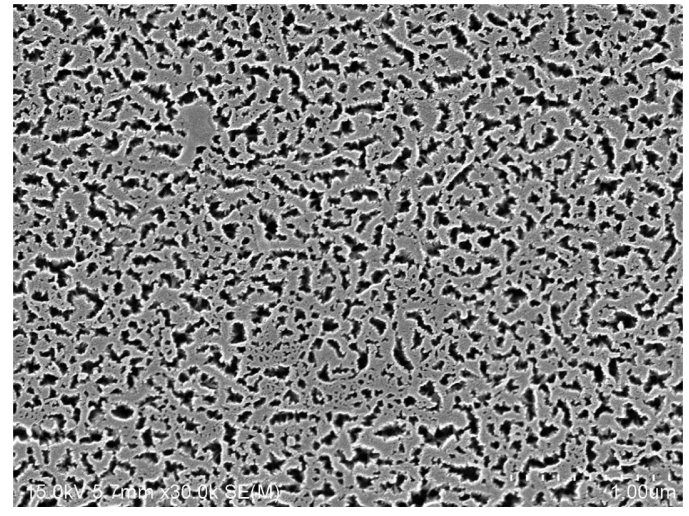

Fig. 4. Surface FE-SEM image of DBR PSi sample.

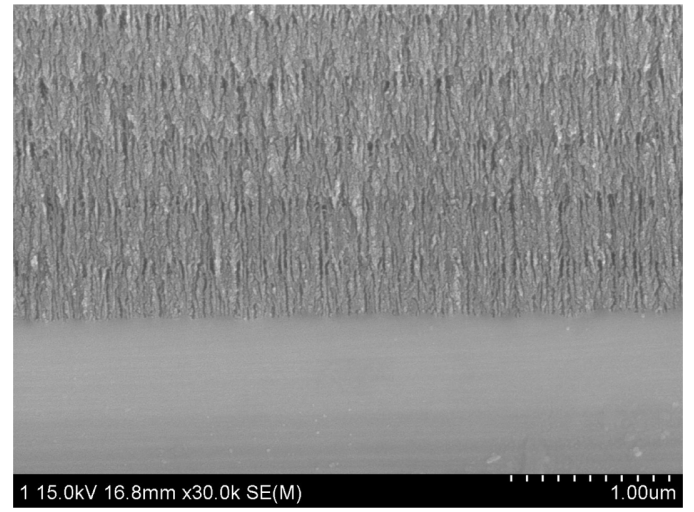

Fig. 5. Cross-section FE-SEM image of DBR PSi sample.

PSi samples prepared with various different high and low applied currents. This result illustrates that the reflection band can be controlled by tuning the etching frequency and appear anywhere in the visible range depending on the applied current densities.

\section{Conclusion}

DBR PSi layers of different characteristics were formed to determine their optical constants in the visible wavelength range. The surface image of DBR PSi indi-

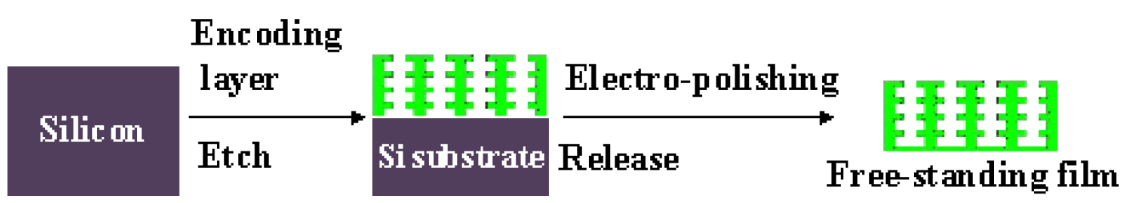

Fig. 6. Schematic diagram for the preparation of free-standing DBR PSi. 


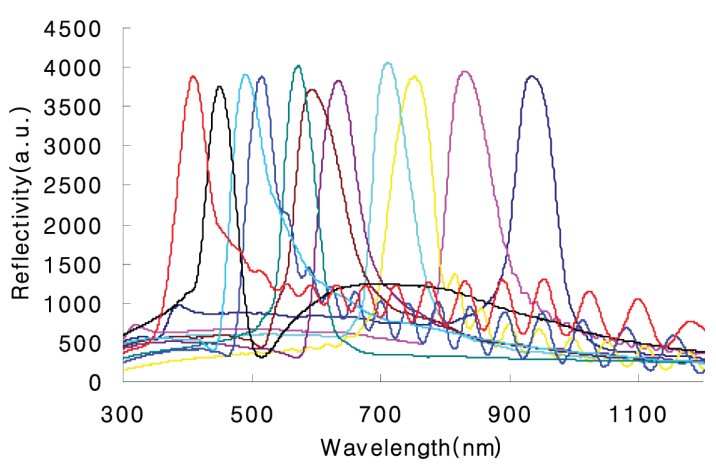

Fig. 7. Reflection spectra of prepared DBR PSi samples.

cates that the even distributions of the etching process results two distinct refractive indices in the contrast. DBR PSi exhibited a porosity depth profile that related directly to the current-time profile used in etch. Freestanding DBR PSi film was obtained by applying an electro-polishing current.

\section{Acknowledgment}

This research was financially supported by the Ministry of Education (MOE) and National Research Foundation of Korea (NRF) through the Human Resource Training Project for Regional Innovation (2012H1B8A 2026282).

\section{References}

[1] B.-Y. Lee, M. Hwang, H. Cho, H.-C. Kim, and S. Jang, "Characterization and surface-derivatization of porous silicon", J. Chosun Natural Sci., Vol. 4, pp. 182-186, 2011.

[2] D. H. Jung, "Biosensor based on distributed Bragg reflector photonic crystals for the detection of protein A", J. Chosun Natural Sci., Vol. 3, pp. 33-37, 2010.

[3] S. H. Jang, "Chemical and physical properties of porous silicon", J. Chosun Natural Sci., Vol. 4, pp. 1-6, 2011.

[4] B.-Y. Lee, M. Hwang, H. Cho, H.-C. Kim, and S. Jang, "Chemical and physical properties of porous silicon", J. Chosun Natural Sci., Vol. 4, pp. 187-191, 2011.
[5] Y. D. Koh, "Analysis on oxidation of porous silica obtained from thermal oxidation of porous silicon", J. Chosun Natural Sci., Vol. 3, pp. 153-156, 2010.

[6] J. M. Han, "Photoluminescence of porous silicon according to various etching times and various applied current densities", J. Chosun Natural Sci., Vol. 3, No. 3, pp. 148-152, 2010.

[7] S. H. Jang, "Study on thickness of porous silicon layer according to the various anodization times", J. Chosun Natural Sci., Vol. 3, pp. 206-209, 2010.

[8] S. Jang, "Investigation of the changes of FabryPerot fringe patterns in porous silicon during etching process", J. Chosun Natural Sci., Vol. 5, pp. 1317, 2011.

[9] B. Kim, "Investigation of relationship between reflection resonance and applied current density in bragg photonic crystal", J. Chosun Natural Sci., Vol. 5, pp. 27-31, 2011.

[10] M. W. Hwang and S. D. Cho, "Detection of organic vapors using change of Fabry-Perot fringe pattern of surface functionalized porous silicon", J. Chosun Natural Sci., Vol. 3, pp. 168-173, 2010.

[11] B.-Y. Lee, M. Hwang, H. Cho, H.-C. Kim, and S. Cho, "Multiple-bit encodings of bragg photonicstructures by using consecutive etch with various square wave currents", J. Chosun Natural Sci., Vol. 4, pp. 192-196, 2011.

[12] S. D. Cho, "Preparation of polystyrene thin films containing Bragg structure and investigation of their photonic characteristics", J. Chosun Natural Sci., Vol. 3, pp. 138-142, 2010.

[13] S. D. Cho, "Detection of nitroaromatic compounds with functionalized porous silicon using quenching photoluminescence", J. Chosun Natural Sci., Vol. 3, pp. 202-205, 2010.

[14] K. S. Jung, "Fabrication and characterization of DBR porous silicon chip for the detection of chemical nerve agents", J. Chosun Natural Sci., Vol. 3, pp. 237-240, 2010.

[15] Y. C. Koh, "1-D photonic crystals based on Bragg structure for sensing and drug delivery applications", J. Chosun Natural Sci., Vol. 4, pp. 11-14, 2011.

[16] S. G. Kim, "Optical characterization of smart dust based on photonic crystals and its sensing applications", J. Chosun Natural Sci., Vol. 4, pp. 7-10, 2011. 